\title{
Langage obscène et injurieux dans La Voyeuse interdite de N. Bouraoui et C'est le soleil qui m'a brûlée de C. Beyala
}

\section{Obscene and Offensive Language in La Voyeuse Interdite by N. Bouraoui and C'est le soleil qui m'a brûlée by C. Beyala}

\author{
Assia MARFOUQ ${ }^{1}$ (i), Abdelghani BRIJA² (])
}

${ }^{1}$ Prof., Hassan First University, Laboratory of Didactic Engineering, Entrepreneurship, Arts, Literature and Languages (LIDEALL), Settat, Morocco

${ }^{2}$ Prof., Mohamed V University of Rabat, Morocco

ORCID: A.M. 0000-0002-1803-3279; A.B. $0000-0002-3355-3395$

\section{Corresponding author:}

Assia MARFOUQ

Hassan First University, Laboratory of Didactic Engineering, Entrepreneurship, Arts, Literature and Languages (LIDEALL), Settat, Morocco

E-mail: assia.marfouq@uhp.ac.ma

Submitted: 22.01 .2021

Revision Requested: 05.04.2021 Last Revision Received: 12.04.202 Accepted: 06.09.2021

Citation: Marfouq, A., \& Brija, A. (2021) Langage obscène et injurieux dans $L a$ Voyeuse interdite de N. Bouraoui et C'est le soleil qui m'a brûlée de C. Beyala. Litera, 31(2), 653-666.

https://doi.org/10.26650/LITERA2021-866664

\begin{abstract}
RÉSUMÉ
La sexualité est une thématique très prisée dans la littérature africaine de manière générale. Fortement liée à d'autres thèmes comme le patriarcat, l'émancipation de la femme, la domination masculine, etc., la sexualité traduit le conflit de genre en la femme et l'homme et trahit l'idéologie masculine dans les sociétés africaines. La sexualité dans le corpus choisi pour notre étude, à savoir C'est le soleil qui m'a brûlée de Calixthe Beyala et La Voyeuse interdite de Nina Bouraoui, est intimement liée au projet d'écriture de ces auteurs qui ont choisi d'opter pour le langage obscène et les injures afin de montrer à quel point l'homme réduit la femme à un simple objet sexuel et afin d'adopter une position de contre-attaque dans le souci de réduire l'homme à son organe sexuel. Le langage obscène devient alors un moyen de proclamation de la liberté des femmes. Les auteures utilisent aussi ce langage choquant afin de réveiller les consciences des lecteurs sur l'urgence d'établir l'équilibre dans les relations entre femme et homme. La lecture du langage obscène et injurieux à travers les deux romans africains soumis à l'étude nous permettra de voir comment les auteurs dépeignent sans retouche des scènes sexuelles et les remettent constamment dans leur cadre idéologique et social.
\end{abstract}

Mots-clés: Langage, sexualité, trivialité, Beyala, Bouraoui

\section{ABSTRACT}

Sexuality is a very popular theme in African literature in general. Strongly linked to other themes like patriarchy, female emancipation, male domination, etc., sexuality translates the gender conflict in women and men and betrays male ideology in African societies. Sexuality in the corpus chosen for our study: C'est le soleil qui m'a brûlée by Calixthe Beyala and La Voyeuse interdite by Nina Bouraoui, is intimately linked to the writing project of these authors who chose to opt for obscene language and insults in order to show to what extent man reduces woman to a simple sexual object and in order to adopt a position of counterattack in the concern to reduce man to his sexual organ. Obscene language then becomes a means of proclaiming the freedom of women. The authors also use this shocking language to awaken readers' awareness of the urgent need to establish a balance in the relationship between women and men. Reading the obscene and offensive 
language throughout the two African novels submitted for study will allow us to see how the authors portray sexual scenes without retouching and constantly put them back into their ideological and social framework.

Keywords: Language, sexuality, triviality, Beyala, Bouraoui

\section{EXTENDED ABSTRACT}

The question of sexuality is omnipresent in African literature. The presence of woman in the African novel is certainly vital, because she represents the most significant and interesting character. Her body and sex have become a subject of writing and a romantic object that assume thematic and ideological interpretations.

In order to assert the presence of sex as a means of proclamation, writers refuse to be silent or to hide behind words. Approaching sex is a way of speaking freely and openly by confronting what is usually secret and hidden, that is to say forbidden, to be verbalized at the risk of overturning and breaking the stability of social order. The theme of sexuality has given birth to novelists who violate prohibitions and taboos and venture into writing that is intentionally shocking.

Disobeying the patriarchy and stereotypes of society which make woman a simple sexual object to be consumed according to the desire of man becomes the weapon of any African woman who wishes to change her situation of damned and submissive in order to access emancipation and freedom of choice. The silence imposed by men in African Maghreb and sub-Saharan societies with the aim of subjecting women to enslavement is a subject much addressed by gender studies and current postcolonial studies which further clarify the nature of the link between men and women, particularly in formerly colonized countries. This situation does not seem to be able to last over time, especially after the emergence of female writing.

The theme of sexuality and the use of trivial language particularly interested the African Maghreb and sub-Saharan authors. As a result, we have chosen to examine two representative novels from the Maghreb and sub-Saharan Africa. We will be particularly interested in two writers representative of this theme in both Maghreb and Sub-Saharan spaces: Cest le soleil qui me brûlée by Calixthe Beyala and La Voyeuse interdite by Nina Bouraoui. These authors talk about sex without restraint, describing it in a blunt way. They approach sex by portraying it in a strong, detailed, and unedited way in erotic or even sometimes pornographic-looking scenes. Their concern is to stage carnal 
relationships bathed in deviation, prohibition, transgression, disorder, and liberation. Our work consists of a careful analysis of coarse language and the lexicon that dominates it while constantly linking with patriarchal and feminist ideology.

The use of trivial language is far from free in the novels submitted to our review. We will see how this language is indeed part of a logic that is part of the authors' writing project based essentially on excess, excessiveness, and hyperbole in order to awaken the consciousness of the readers and to raise awareness of social debauchery and the flagrant imbalance between men and women. We will show that the exposure of social reality through trivial and pornographic language also signifies to what extent the relationship between man and woman in African societies have a sexual nature which reduces women to a simple object of pleasure. The authors did not fail to respond to this inferiorization imposed by man with demeaning and reductive language. We will also see how the offensive interpellation metaphorically translates feminine attitudes and their perceptions of the masculine gender. It is a way of imposing the subjugation that man knows how to impose on woman. The identification of man by repeatedly derogatory questioning reflects how dominant ideology is through language and has the power to exclude the subject. The strategies of language attack and the defense undertaken by men and women show that even language can be subjected to an ideology which structures it and prepares it for the destruction of a gender, a class, or a race. 


\section{Introduction}

Le sexe est libéré de tout tabou d'ordre social. Dans le cas des romans africains, il s'agit de l'imagination sans limites des écrivains africains de la nouvelle génération. On le trouve partout dans ces textes : dans la nudité, le dévoilement et l'exhibition. II est le moteur d'articulation et de dynamisation de la fiction romanesque.

La crudité des mots des auteures soumises à l'étude et le « libertinage » textuel veulent sans aucun euphémisme dévoiler à la fois toute débauche sexuelle et tout désordre social, mais aussi lever le voile sur le malaise et la désorientation vécues par les sociétés modernes qui vivent sans repères et dans un désordre total.

Le roman contemporain est en effet caractérisé par une certaine tendance excessive de transgresser les tabous et les interdits. La débauche sexuelle dans le texte romanesque est alors une image de la débauche sociale que les auteurs étalent et exposent en public de manière isolée avec une intention manifeste de choquer le lecteur et de représenter le vécu de manière figurée en mettant le doigt sur sa laideur sans aucune triche ou jeu hypocrite avec les mots et les expressions par souci de conformisme ou de convenance. II n'est pas du tout étonnant de remarquer que les textes africains sont remplis de corps, de chair, de sexe et de sexualités débridées et désordonnées.

La présente étude propose d'étudier un aspect particulier de la sexualité dans le roman africain à travers le langage obscène et injurieux qui marque les sujets et influe leur identité. Notre analyse se fera sur un corpus constitué de deux romans représentatifs de notre thème d'étude et des notions sur lesquelles il s'articule comme le patriarcat, le pouvoir, l'idéologie et l'assujettissement : La Voyeuse interdite de Nina Bouraoui et C'est le soleil qui m'a brûlée de Calixthe Beyala. A la lumière de théories de langage et leurs relations avec le pouvoir et l'idéologie, nous analyserons suivant une approche comparée notre corpus afin de montrer comment l'injure, l'interpellation et le langage obscène contribuent à soumettre la femme et la dévaloriser. Althusser, Foucault, Austin, Rastier et Butler sont nos principaux auteurs qui alimenteront le cadre théorique de notre étude.

\section{Les mots triviaux du langage sexuel}

Dans C'est le soleil qui m'a brûlée de Beyala le discours du sexe et de la sexualité s'affirme comme une manière de lutter contre les vices de la société, particulièrement 
les dépravations sexuelles et les violences contre les femmes. Étaler ces violences dans le texte est une manière de mieux les faire voir, car mettre la réalité à nu permet de bien en prendre conscience. Beyala opte pour un effet de grossissement, et de carnavalisation dans ses textes. On remarque que le processus de carnavalisation permet de voir la vie et le monde à l'envers où tabous et interdits sont mis entre crochets et tout le reste est permis. Ce renversement de situations et de l'ordre établi est une manière qui mène vers la libération, le défoulement et la catharsis.

L'utilisation du langage trivial, grossier et vulgaire n'est pas donc gratuite dans le texte de Beyala ou tout simplement provocateur : il porte toute la signification de son projet romanesque. Les grossièretés présentes dans le texte, les scènes obscènes ainsi que toute transgression exagérée des règles et des normes sociales relèvent de l'écriture carnavalesque caractérisée par l'aspect outrancier. Ce dernier a pour objectif de réveiller la conscience du lecteur et fait référence directement à la débauche qui caractérise réellement la société africaine.

Ainsi, on remarque que les valeurs de l'humanité subissent le renversement, le piétinement et le bafouage. Ce constat est facilement perceptible au niveau du langage. II se ressent à travers un langage qui est à l'image de la réalité, qui y adhère et la restitue. Faire vrai, et être vraisemblable, suppose cette vulgarité au niveau du langage afin de rompre avec toute menterie ou mystification de l'ordre social. Les romancières du sexe traduisent sans détour ou faux-fuyant une réalité sociale qu'il faut absolument mettre à nu.

L'écriture carnavalesque retrouvée dans le texte beyalien permet en outre de responsabiliser le lecteur en mettant devant ses yeux les hypocrisies cachées derrière l'ordre social. Par conséquent, on remarque que derrière la crudité des mots qui choquent et déstabilisent, derrière le langage du corps et du sexe, se cache une violente satire sociale où les mots orduriers et triviaux sont rendus banals tant la perversion et le vice a franchi depuis très longtemps le seuil de l'acceptable.

Les écrivaines africaines francophones recourent à un langage obscène, adjectif que le Dictionnaire Le Petit Robert définit comme « tout ce qui blesse ouvertement la pudeur par des représentations d'ordre sexuel ». Les récits de notre corpus présentent en effet une offense aux règles de bienséance à travers les grossièretés, la sexualité et même la scatologie afin de représenter le monde chaotique des sociétés africaines. À 
titre d'exemple, Beyala procède par des descriptions obscènes érotiques et pornographiques.

En effet, la référence au sexe émaille constamment le roman beyalien. Tout d'abord, Ateba raconte une scène sexuelle qui remonte au temps de son enfance. Il s'agit d'une expérience initiatique qu'Ateba a vécue avec le petit Gon, le fils de Madame Combi. Cette scène est décrite avec beaucoup de détails et avec des mots soigneusement choisis, de sorte que le lecteur a l'impression de témoigner de près de cette scène romanesque:

Elle [Ateba] prend le sexe dans ses mains. Elle le serre. Il gonfle. Elle s'allonge. Elle relève sa robe. Elle invite Gon à grimper. Le petit sexe tendu se frotte au hasard dans son vagin, très vite, un peu à la manière des coqs. Quelquefois il vise juste. « Non, pas là, crie-t-elle. Et le petit sexe repart, alerte, prêt à conquérir les recoins permis ». (Beyala, 1999, p. 66)

On remarque que le langage cru et obscène est véhiculé inexorablement à travers le langage corporel féminin. L'érotisme investit massivement le texte beyalien à tel point que nous pouvons avancer, en utilisant les termes de Jean DÉjeux que l'auteure jouit « à farfouiller avec démence au-dessus de la ceinture » (Saigh Bousta, 1989, p. 64). Il est intéressant de remarquer que la vision réciproque entre homme et femme est construite sur le sexe et dite dans le texte beyalien à travers le langage de la sexualité. BEYALA opte pour une poétisation du corps en évoquant la manipulation que les hommes en font. L'auteure se moque de l'homme par le biais de son organe sexuel: « J'entame la chanson de l'homme qui veut que sa valeur se reconnaisse à la longueur de son sexe et sa qualité à l'absence de prépuce. » (Beyala, 1999, p. 40) et évoque sa vision réductrice de la femme par l'évocation de l'acte sexuel : « Bouge pas et baise. » (Beyala, 1999, p. 64), expression qui renvoie directement à l'infantilisation de la femme qu'on peut facilement comparer à « tais-toi et mange » que l'on adresse à un enfant.

Dans La Voyeuse interdite de Bouraoui, la même idée de la description réductrice de l'homme à l'égard de la femme s'impose. Fikria nous livre ainsi une scène sexuelle entre son père et sa génitrice :

J'ai vu mon père s'acharner sur ces deux grosses mamelles pleines de veines (...), sa bouche (...) essayait d'engloutir une des poches nerveuses 
de ma génitrice, mais il la recrachait aussitôt, ses deux mâchoires ne pouvant contenir la totalité du morceau de chair (...) mon père (...) coincé entre les cuisses lourdes et peu agiles de ma mère, il s'agrippait tant bien que mal à cette chaloupe beuglant comme un animal traqué. Tout petit, tout fin, tout noir, il ressemblait à un vers creusant son trou dans une terre aride et buissonneuse, pour lui, la garrigue s'agitait et enfonçait ses épines vénéneuses dans le bout de chair découvert. (...) la victime replia ses cuisses monstrueuses. Ses cheveux étalés sur le carrela, comme une tête de loup ébouriffée par la poussière, dessinaient le corps d'un oursin ouvert. (Bouraoui, 1991, pp. 36-37)

Contrairement au langage beyalien où l'auteure s'exprime avec des mots précis qui renvoient directement au champ lexical de la sexualité, nous remarquons que BOURAOUI métamorphose la vision que porte I'homme sur la femme, mais différemment. Car au lieu de décrire l'acte sexuel par un lexique qui renvoie sans détour aux organes génitaux et aux actions des deux partenaires, l'auteure se soustrait à tout cela et aborde la question sur un plan métaphorique. Ainsi, les sémèmes « animal », « terre », « aride », « buissonneuse », « garigue », « épines », « vénéneuses », « loup », « oursin », « vers », « creusant », « trou » renvoient aux référents de la faune et de la flore créant ainsi une « impression référentielle » (Rastier, 1987, p. 87) comme l'appelle François Rastier qui nous fait penser au mode de vie dans une véritable jungle. Rappelons à ce sujet ce que déclare Rastier à propos de l'impression référentielle :

Dans le cadre d'une sémantique intensionnelle, le problème du "correspondant" d'un contenu dans le "monde naturel" est malaisé voire impossible à poser, puisqu'il se ramène en somme à celui de la dénotation ou, si l'on préfère, de la référence. On montrera que l'impression référentielle produite par un énoncé ou un prédicat est fonction des relations entre les contenus de cet énoncé (ou de ce prédicat) et de domaines sémantiques socialement normés. (Rastier, 1987, p. 87)

Ce croisement de références qui mélange la vie sexuelle à la vie bestiale laisse entrevoir un point de ressemblance crucial : la loi du plus fort. Par conséquent, dans un rapport sexuel comme dans la forêt, il n'y a pas lieu pour l'égalité ou au partage, plutôt des rapports entre un fort et un faible, un dominant et un dominé. La femme joue le rôle de proie dans l'énoncé, car elle est qualifiée de « victime ». 
La narratrice dans C'est le soleil qui m'a brûlée use d'un langage cru qui manipule aisément le vocabulaire érotique. Son objectif est de lever tous les interdits devant la voix féminine et nier l'homme. Celui-ci est montré comme un être très réduit dont la virilité subit le pire mépris et est tournée à la dérision. Beyala recourt à l'agression verbale à l'encontre de l'homme et son corps qu'elle malmène en le considérant comme oppresseur. On retrouve chez elle des expressions telles que « sexe imbécile » (Beyala, 1999, p. 98) pour désigner l'organe phallique masculin, « la sève inutile » (Beyala, 1999, p. 54) qui en découle, « cette flèche qui pointe de son bas ventre » (Beyala, 1999, p. 131), «fesses comme deux demi-cocos » (Beyala, 1999, p. 131), « gombo plié gluant » (Beyala, 1999, p. 76), « truc tout petit et tout rouge » (Beyala, 1999, p. 43). Parallèlement, la narratrice de La Voyeuse interdite de Bouraoui utilise des expressions qui réduisent le pouvoir sexuel de l'homme. Fikria assimile son père à « un vers » qui « creuse son trou » et caractérise son sexe par les adjectifs « fin », « noir » et " petit » et l'assimile à « une arme cachée » (Bouraoui, 1991, p. 37).

\section{Interpellation et injure}

Au registre érotique truffé de mots crus et d'obscénités, on remarque que les écrivaines procèdent par des insultes d'ordre génital et un vocabulaire injurieux et impudique. Avant d'illustrer ce point par des exemples tirés de notre corpus, il nous semble nécessaire de s'attarder sur la définition du sens de l'interpellation injurieuse et son assujettissement de la femme.

Louis Althusser définit l'interpellation comme étant le processus de nommer un individu et à travers lequel ce dernier existe en tant que sujet. Le sujet interpellé acquiert une identité par le biais de l'adresse d'un locuteur. II n'existe en effet qu'à travers le nom que les individus lui attribuent. Selon Althusser, l'interpellation permet de passer de l'individualité à la subjectivité et confère une identité et une existence sociale à l'individu. Althusser et Foucault ont suffisamment abordé la question de l'interprétation dans son rapport avec le sujet, le pouvoir et l'assujettissement. Pour Althusser, il affirme que « toute idéologie interpelle les individus concrets en sujets concrets, par le fonctionnement de la catégorie de sujet » (Althusser, 1976, p. 113) et que "L'individu interpellé se retourne. Par cette simple conversion physique de 180 degrés, il devient sujet » (Althusser, 1976, p. 113). Pour Foucault, il faut étudier « la manière dont un être humain se transforme en sujet » (Foucault, 1975, p. 298). 
L'interpellation suscite un émetteur ou un énonciateur et un récepteur. L'émetteur est obligatoire dans cette opération, car sans l'intervention de cet agent, l'interpellation ne peut avoir lieu. En interpellant, l'émetteur donne un statut social au récepteur, ce qui signifie que l'énonciateur est toujours en position de force et de domination. En interpellant, l'énonciateur mobilise une idéologie, des conventions et des contraintes. Ces dernières œuvrent pour assigner au destinataire un statut, un rôle, une place. En un mot, le produit langagier de l'émetteur résulte de toute une société et non pas d'un seul individu. L'interpellation est alors un langage métaphorique qui traduit nos perceptions et nos attitudes face à la race, la classe et le genre.

L'interpellation contribue à identifier les individus d'une part, mais les rend assujettis à une idéologie. Dans Positions, Althusser affirme que «l'individu est interpellé en sujet pour qu'il se soumette aux ordres du Sujet, donc pour qu'il accepte son assujettissement » (Althusser, 1976, p. 121).

Dans Le Pouvoir des mots, Judith Butler définit l'interpellation de la manière suivante:

L'existence sociale du corps est d'abord rendue possible par son interpellation à l'intérieur des termes du langage. Pour le comprendre, il nous faut imaginer une scène impossible, celle d'un corps qui n'a pas été socialement défini, un corps auquel, à rigoureusement parler, nous n'avons pas accès, et qui néanmoins devient accessible à l'occasion d'une adresse, d'un appel, d'une interpellation qui ne le «découvre» pas, mais qui, le constitue. (Butler, 2006, p. 26)

Butler poursuit sa définition en liant l'interpellation à l'assujettissement qu'elle provoque chez le sujet :

L'interpellation est un acte de discours dont le «contenu» n'est ni vrai ni faux : décrire n'est pas son objectif premier. Son but est de désigner et d'établir un sujet assujetti, de produire ses contours sociaux dans le temps et l'espace. (Butler, 2006, p. 56)

Pour que l'individu puisse exister socialement, il serait donc contraint d'accepter sa dépendance à l'adresse avec laquelle il est interpellé. Être interpellé signifie que l'on est désigné par un simple nom ou une injure qui nous donnent une place que 
nous n'avons pas choisie. Butler estime que l'acte de l'interpellation, sans qu'elle soit injurieuse, implique un paradoxe selon lequel l'interpellation constitue à la fois le sujet et contribue à son assujettissement : « l'acte même de l'interpellation nous inflige une « injure », puisqu'il interdit la possibilité de l'auto-genèse du sujet » (Butler, 2006, p. 50). Il s'agit d'une subordination qui s'opère sur deux plans : accepter l'interpellation et être partie intégrante d'une société où les individus sont reconnus et reconnaissables et être soumis à un pouvoir qui précède l'existence de l'individu et dépasse son pouvoir.

Si l'interpellation désigne le sujet et l'identifie, on remarque qu'elle se cristallise dans notre corpus dans un langage agressif et flagrant. Son agressivité vient du fait qu'il ne s'agit pas d'une désignation banale des personnages, mais bien de l'utilisation du lexique péjoratif, impudique et rattaché principalement à l'organe génital, à la scatologie et aux mots orduriers. Les termes dégradants dans lesquels sont interpellés les personnages féminins dans C'est le soleil qui máa brûlée de Beyala sont particulièrement des mots tels que "Putain », "Garce », "Guenon ». Dans La Voyeuse interdite de Bouraoui la femme est qualifiée de « poufiasse » (Bouraoui, 1991, p. 98), de « souillure » (Bouraoui, 1991, p. 61), de «Fille, fourre, femme, fornication, faiblesse, flétrissures » (Bouraoui, 1991, p. 31). Ces noms semblent attaquer directement la femme, son corps et sa sexualité et rappeler à la femme son statut d'infériorité dans la société et sa marginalisation. Le mot « putain » à titre d'exemple est une séquence langagière qui donne à la femme l'identité de prostituée et la réduit à un statut social inférieur et dévalorisant. De ce fait, nous estimons que l'acte d'interpellation injurieuse n'est nullement un acte uniquement illocutoire, car il produit un effet instantané sur la personne nommée et devient dès lors un acte à la fois illocutoire et perlocutoire. Butler précise que «Si l'on peut en ce sens dire qu'un mot « fait » une chose, c'est donc que le mot ne se contente pas de signifier une chose, mais que cette signification est aussi une réalisation de la chose -interpellation-assujettion » (Butler, 2006, p. 72). L'acte illocutoire contenu dans l'appellation injurieuse à travers le lexique dévalorisant donne lieu à un acte perlocutoire vu les sentiments et les pensées que produit le discours injurieux sur l'injurié. Parmi les « conditions de réussite » (Austin, 1991, p. 97) de l'interpellation selon Austin est la place privilégiée du locuteur à laquelle nous rajoutons le fait que l'interpellation injurieuse fait preuve de répétitions et concerne toujours un seul aspect afin de mieux s'imposer. Selon Austin, l'injure est dotée d'une dimension pragmatique : «l'injure possède toujours une force illocutoire voulue par celui qui l'énonce » (Austin, 1991, p. 102). 
Dans notre corpus, nous constatons à cet effet que le lexique injurieux et impudique le plus fréquent est rattaché à la sexualité féminine et ses organes génitaux. Ainsi, les termes «Pute », « Garce » et « une vient-me-baiser » produisent le dégoût et rejettent la femme sexuellement parlant. La femme éprouve certainement de la honte à l'égard de ces énoncés et se sent aliénée. L'appellation « pute » ou « putain » évoquée abondamment dans les romans de notre corpus se présente sous la forme d'un fragment langagier dont le sens est lourdement chargé par la dimension historique qui le rend imposant comme le souligne Butler :

Les noms injurieux ont une histoire, laquelle est invoquée et renforcée au moment de leur énonciation, mais ce n'est pas explicitement formulée. Ce n'est pas seulement l'histoire de leurs usages, de leurs contextes et de leurs buts. C'est la manière dont ces histoires se sont inscrites et arrêtées dans le nom et par lui. Le nom a ainsi une historicité qui peut être comprise comme l'histoire devenue antérieure ou non, qui en est venue à constituer la signification contemporaine du nom. La sédimentation de ses usages qui ont été assimilés par le nom, une sédimentation, une répétition qui se fige et qui donne sa force au nom. (Butler, 2006, p. 59)

De ce fait, l'appellation injurieuse puise toute sa force dans la répétition à travers I'histoire, de la répétition qui colle bien dans la mémoire, de la répétition qui traumatise comme le souligne encore Butler :

Si nous comprenons la force injurieuse comme un effet de son historicité, alors cette force n'est pas le simple effet causal d'un coup infligé, mais elle fonctionne en partie grâce à une mémoire chiffrée ou à un trauma, qui vit dans le langage et est véhiculé par lui. (Butler, 2006, p. 59)

L'interpellation injurieuse est un discours qui au lieu de faire de la femme un sujet, la constitue en tant qu'assujettie, car il la domine, l'exclut et l'oblige à respecter son infériorité imposée par l'idéologie. La langue, véritable véhicule de cette dernière, se trouve soumise à des questions d'ordre sexiste, car elle reflète à titre d'exemple la primauté du masculin comme le remarque cette fois-ci Marina Yaguello :

Ce que révèle l'étude du genre, «grammatical» ou «naturel», et de ses valeurs symboliques, de son fonctionnement (absorbation du féminin 
par le masculin), des dissymétries morphologiques: (les noms d'agent, dénotatives, connotatives), de la langue du mépris (les qualificatifs injurieux pour la femme réduite au choix entre le titre de Madone et celui de Putain, l'argot sexuel et sexiste: c'est le même), de l'identité sociale des femmes (Elles sont toujours définies par le père ou le mari), des dictionnaires enfin, qui sont des créations idéologiques et dont les définitions reflètent la mentalité attardée des usagers de la langue. (Yaguello, 1998, p. 149)

La langue donne l'image de la différenciation sexuelle et apparaît donc comme un véritable véhicule de l'idéologie masculine qui opprime la femme comme le souligne Shoshana Felman :

L'oppression des femmes se détecte non simplement dans le fonctionnement des structures sociales, médicales et politiques, mais dans les présupposés du raisonnement discursif lui-même, dans les mécanismes subtils du procès même de la production du sens. (Felman, 1976, p. 138)

Le lexique du roman beyalien et bouraouien montre clairement les injures inépuisables qui se conçoivent en passant par le corps et le sexe féminins. Dès le bas âge, la fille est traitée par un lexique qui se réfère à sa faiblesse, sa fragilité et la honte qu'elle cause à sa famille et son entourage : «Fille, foutre, femme, fornication, faiblesses, flétrissures » (Bouraoui, 1991, p. 31), alors que le garçon est sensibilisé à sa force et est soutenu par sa famille et son entourage. Cette idée se voit illustrée dans C'est le soleil qui m'a brûlée où l'homme est souvent assimilé à un coq : "Le petit sexe [de Gon] tendu se frotte dans son vagin (...) à la manière des coqs » (Beyala, 1999, p. 66) et la femme est coincée dans le rôle péjoratif de poule, exemple que l'on note à travers la scène du « rite de l'œuf » où les vieilles femmes vérifient la virginité des jeunes filles en introduisant un œuf dans leurs vagins. Cette péjoration qui assimile la femme à une volaille la réduit à un rôle physiologique, celui d'exister pour assurer la reproduction de l'espèce humaine.

Le langage injurieux peut aussi être connoté, c'est-à-dire implicite dans la mesure où un même mot peut renvoyer à deux significations différentes, rien qu'en passant du genre féminin au masculin. Ainsi les adjectifs « vieux » et « vieille » que l'on rencontre fréquemment dans notre corpus désignent des signifiés différents. Un homme vieux est un homme sage dont la vie riche d'expériences est formatrice, alors qu'une femme 
vieille est particulièrement remarquable par son infertilité et la baisse de son pouvoir de séduction, donc son inutilité dans la société.

La femme est souvent désignée par ses organes génitaux de manière dépréciative qui la chosifie et la réduit à une sorte de récepteur de sexe. Ainsi, nous rencontrons dans C'est le soleil qui m'a brûlée des termes comme " trou », " vase », " grotte », " gare » qui se réfèrent au sexe de la femme. La femme se trouve alors diminuée et injuriée à travers ses organes génitaux qui sont décrits comme des lieux ou des objets sales, laids et qui inspirent la honte. Rappelons à cet égard la scène où Jean Zepp a trainé par les cheveux sa maitresse devant le miroir pour qu'elle regarde « son sexe poisseux » (Beyala, 1999, p. 98).

Qu'elle soit mère ou prostituée, la femme semble ne pas échapper au lexique injurieux qui la décrit en tant que « salope », « putain » ou de " garce ». Comme le souligne Pierre Guiraud : « II apparaît que toute femme est une putain en puissance et à ce titre marquée de stigmates de la prostitution : laideur, puanteur, méchanceté » (Guiraud, 1978, p. 99). Cette idée trouve écho dans le fait que toute femme est considérée comme prostituée par les injures «fille de pute » ou «fils de pute » qui visent prioritairement la mère en l'assimilant à une pute. Cet exemple frappant montre encore une fois que le discours injurieux est sexiste, car l'honneur de l'homme semble être injurié à travers celui de la femme. On attaque alors l'homme par l'injure, mais par le biais de la femme. La langue qui semble soumise à l'idéologie masculine et patriarcale exploite la femme comme toile de fond dans sa structure lexicale injurieuse. Remarquons que dans les deux œuvres de notre corpus, les injures sont toujours de genre féminin, car leur lexique admet comme suffixes « ouilles», « aille » ou encore « ure ». Donnons à ce titre l'exemple de « souillure », «flétrissures », « fripouille » et « canaille».

Finalement, nous constatons que le langage interpellatif impudique reflète une pratique qui chosifie la femme, l'assassine et éradique son existence en tant que sujet efficace et valorisé. Les injures qui portent essentiellement sur le corps féminin et sa sexualité montrent une certaine angoisse chez le sujet masculin qui essaye de tisser dans la toile de la langue un discours réducteur de la femme, véritable témoin de sa puissance. L'homme procède à la stratégie de l'attaque par le biais du langage afin d'empêcher la femme de monter sur la surface et de le concurrencer en termes de pouvoir et de domination. 
Approbation du comité d'éthique : L'approbation du comité d'éthique n'est pas requise pour ce travail.

Évaluation : Évaluation anonyme par des pairs extérieurs.

Contributions d'auteurs : Conception de travail- A.M., A.B.; Acquisition de données- A.M., A.B.; Analyse/Interprétation des données- A.M., A.B.; Rédaction de manuscrit- A.M., A.B.; Révision critique du manuscrit- A.M., A.B.; Approbation finale et responsabilité- A.M., A.B.

Conflit d'intérêts : Les auteurs n'ont aucun conflit d'intérêts à déclarer.

Subvention : Les auteurs n'ont reçu aucun soutien financier pour ce travail.

Ethics Committee Approval: Ethics committee approval is not required for this study.

Peer-review: Externally peer-reviewed.

Author Contributions: Conception/Design of Study- A.M., A.B.; Data Acquisition- A.M., A.B.; Data Analysis/Interpretation- A.M., A.B.; Drafting Manuscript- A.M., A.B.; Critical Revision of ManuscriptA.M., A.B.; Final Approval and Accountability- A.M., A.B.

Conflict of Interest: The authors have no conflict of interest to declare.

Grant Support: The authors declared that this study has received no financial support.

\section{Bibliographie}

Althusser, L. (1976). Positions. Paris : Les Éditions sociales.

Althusser, L. (1976). « Idéologie et appareils idéologiques d'Etat » in Positions.

Austin, J. L. (1991). Quand dire c'est faire. Paris : Seuil.

Beyala, C. (1999). C'est le soleil qui m'a brûlée. Paris : J'ai lu.

Bouraoui, N. (1999). La Voyeuse interdite. Paris : Gallimard.

Butler, J. (2006). Le Pouvoir des mots. Paris : Éditions Amsterdam.

Felman, S. (1976). La Folie et la chose littéraire. Paris : Seuil.

Foucault, M. (1975). Surveiller et punir. Paris : Gallimard.

Guiraud, P. (1978). Dictionnaire historique, stylistique, rhétorique et étymologique de la littérature érotique. Paris : Payot.

Rastier, F. (1987). Sémantique interprétative. Paris : PUF.

Saïgh Bousta, R. (1989). « Polysémie et motricité discursive : pour une lecture de Harrouda », in Nouvelles du Sud, $\mathrm{n}^{\circ}$ 11, Silex, mai-juin.

Yaguello, M. (1998). Les Mots et les femmes, Essai d'approche sociolinguistique de la condition féminine. Paris : Payot. 